\title{
Rhinosporidiosis with musculoskeletal manifestation: A case series
}

\author{
Bikram Keshari Kar ${ }^{1}$, Rajat Banchhor ${ }^{2, *}$, Rudra Pratap Singh Thakur ${ }^{3}$, Debajyoti Mohanty ${ }^{4}$, Niza Monga ${ }^{5}$ \\ ${ }^{1}$ Associate Professor, ${ }^{2,3}$ Senior Resident, ${ }^{4}$ Additional Professor, ${ }^{5}$ Post Graduate Resident, ${ }^{1-4}$ Dept. of Orthopaedics, ${ }^{5}$ Dept. of \\ Microbiology, All India Institute of Medical Sciences, Raipur, Chhattisgarh, ${ }^{5}$ SMS Medical College, Jaipur, Rajasthan, India
}

\section{*Corresponding Author: Rajat Banchhor}

Email: rajat_banchhor@yahoo.co.in

\begin{abstract}
Rhinosporidiosis is a chronic granulomatous disease caused by Rhinosporidium seeberi. It commonly affects young males and involves the mucous membranes of the nose, nasopharynx and eyes. Involvement of extranasal sites including bone has been rarely reported. Involvement of bone without associated mucocutaneous lesions is extremely rare in rhinosporidiosis. We report a case series of three rare cases of Rhinosporidiosis affecting the musculoskeletal system, involving the clavicle, distal interphalangeal joint of little finger, and lower limb Sartorius muscle.
\end{abstract}

Keywords: Rhinosporidiosis, Phalanx, Distal interphangeal joint, Clavicle, Sartorious, Soft tissue.

\section{Introduction}

Rhinosporidiosis is a chronic granulomatous disease of nasopharynx, oropharynx, conjunctiva, rectum and external genitalia. Floor of the nose and inferior turbinate are the most common sites. This disease is endemic in Chhattisgarh, South India, Sri Lanka and Africa with a prevalence rate of $1.4 \%$. Male to female ratio is $1: 4$ and most cases belong to $15-40$ years of age. It is presumed to originate from stagnant water pools or aquatic life. Traumatic inoculation from one site to others is common. In rare cases it also involves musculoskeletal system of the body after dissemination.

\section{Clinical Presentation \\ Case 1}

A 35 year old woman presented with a 5 month history of swelling and an ulcerating and fungating mass in the anteromedial aspect of the right forearm and right little finger. There was serosanguinous foul smelling discharge from the swelling, which bled on touch. The mass was $9 \times 7 \times 5 \mathrm{~cm}$ in dimension over right little finger, firm, friable, and multilobulated, with a delineated margin and the swelling was of $6 \times 6 \times 4 \mathrm{~cm}$ in dimension over right forearm near elbow (Fig. 1, 2).There was no pain over the swelling and no fever. The patient also had multiple masses over face, abdomen and back (Fig. 3). The nose and oral cavity did not reveal any lesions. The patient had a history of nasal rhinosporodiosis in her childhood for which she had been operated. The serological test for human immunodeficiency virus was negative. Radiography revealed a soft tissue shadow involving areas around right elbow and middle and distal phalnx of right little finger involving distal interphalangeal joint. (Fig. 4)

This case was managed with ray amputation of right little finger from shaft of $5^{\text {th }}$ metacarpal and excision of swelling from subcutaneous plane right forearm along with other cutaneous lesions. (Fig. 5, 6) Histopathology report showed sporangia of various shapes and sizes representing different stages of maturation.

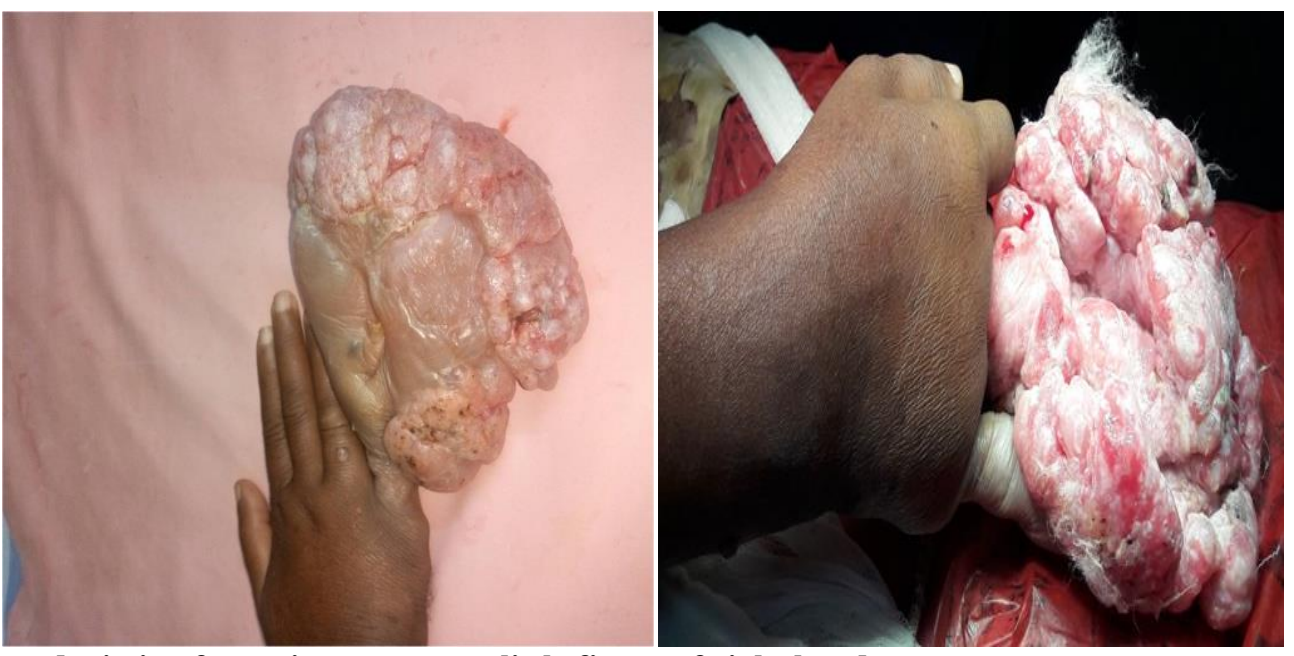

Fig. 1: Picture depicting fungating mass over little finger of right hand 

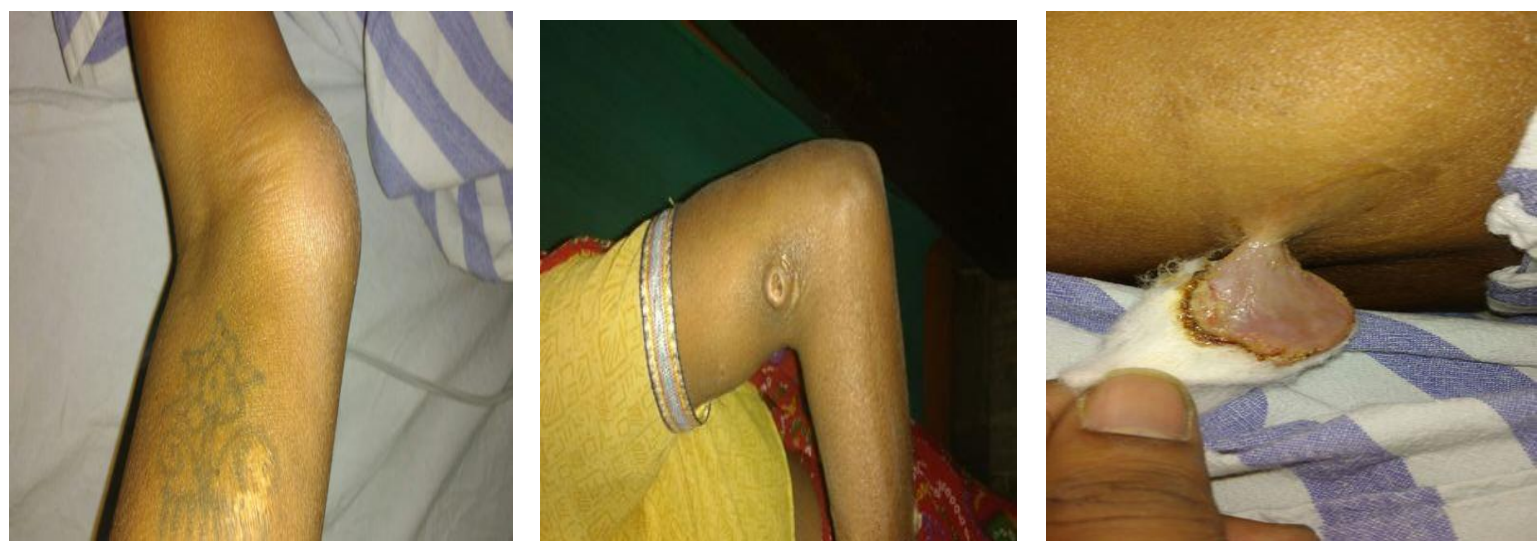

Fig. 2: Musculoskeletal swelling of forearm
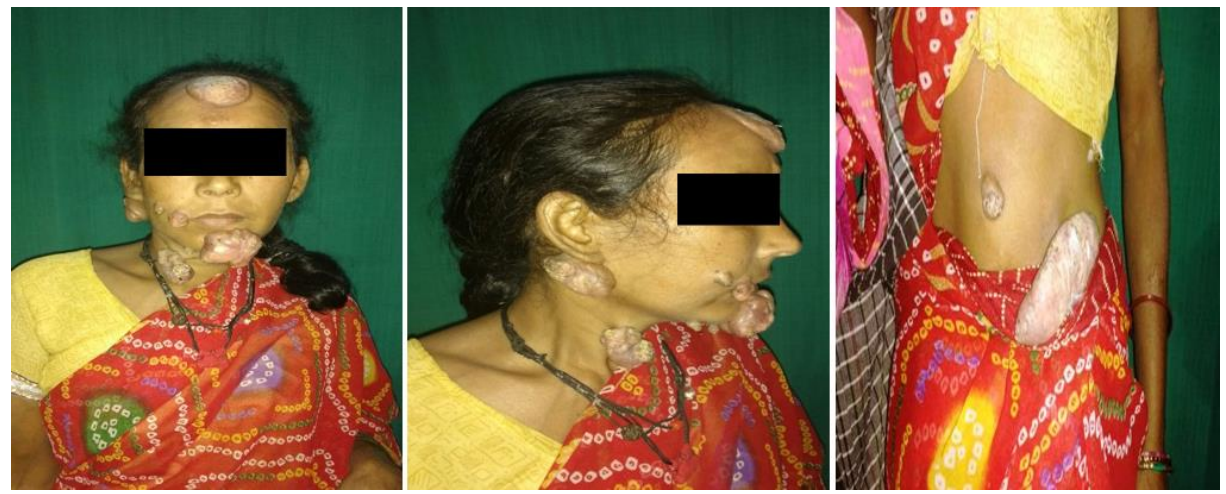

Fig. 3: Clinical picture depicting face and abdomen with multiple swelling with ulceration
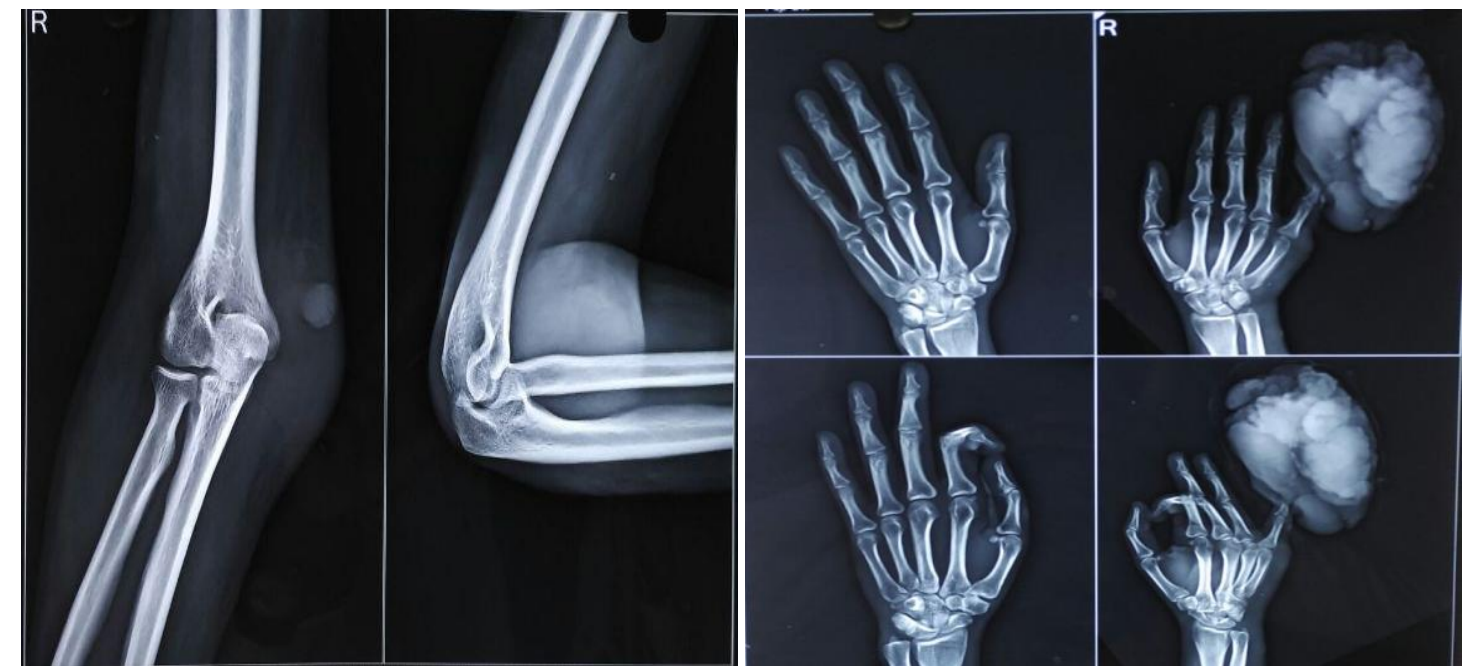

Fig. 4: Radiograph showing osseous involvement and soft tissue shadow 

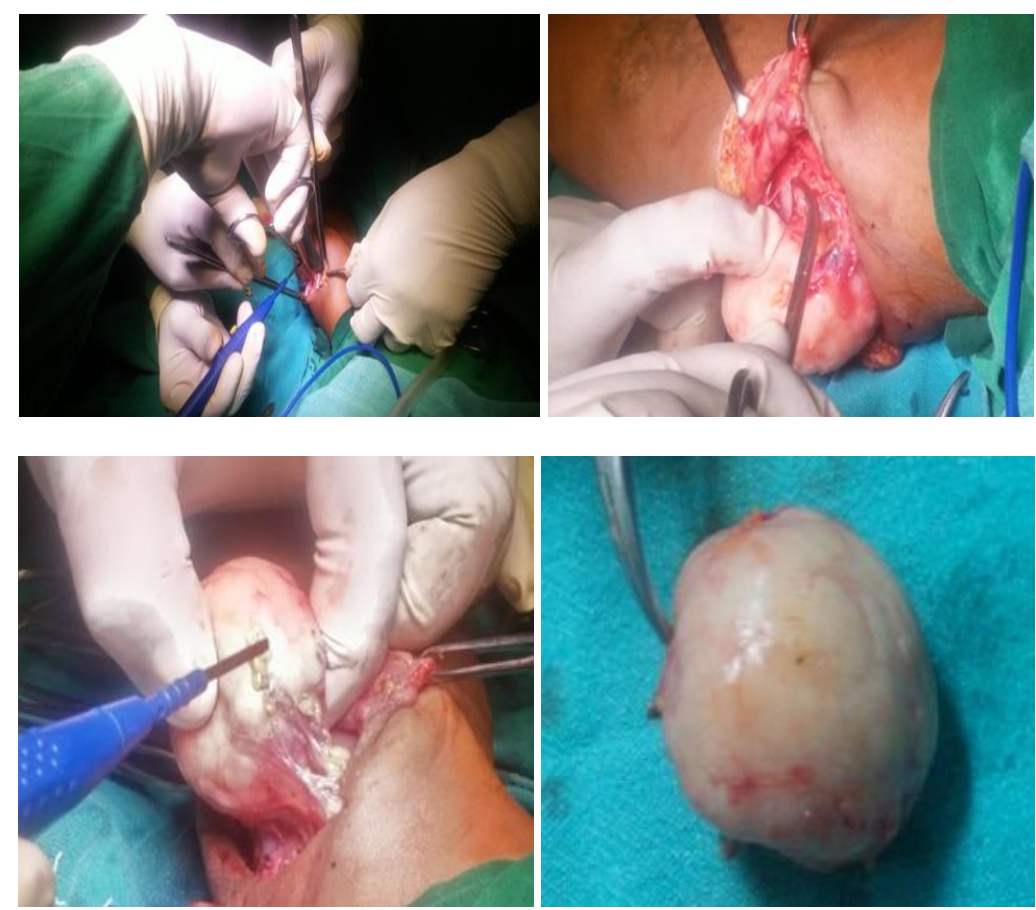

Fig. 5: Excisied cutaneous mass from right forearm
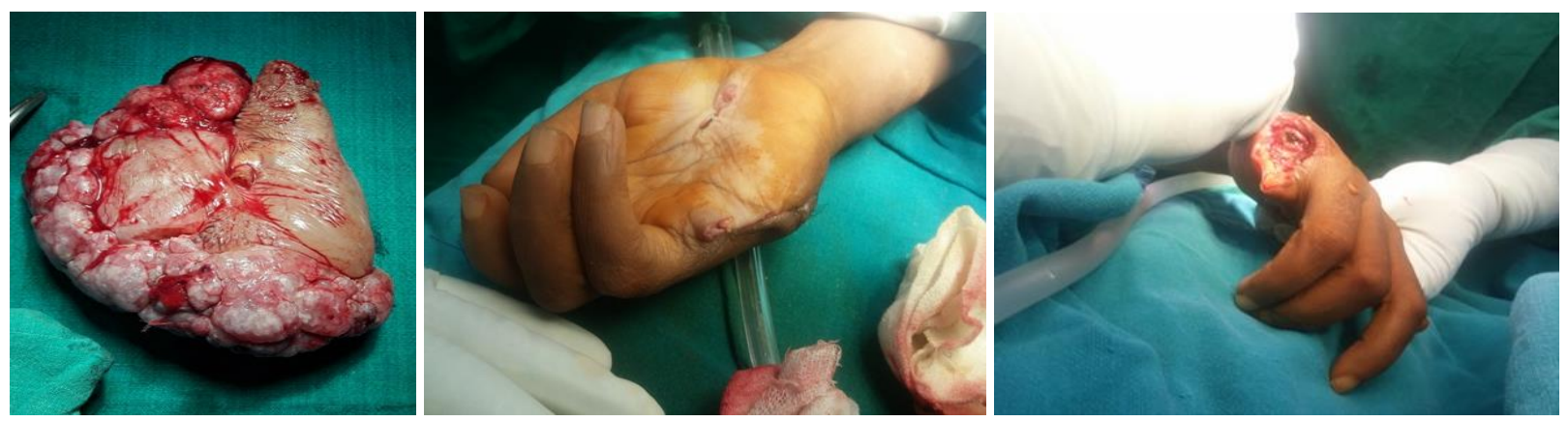

Fig. 6: Excised fungating mass along with right littlr finger from right hand

\section{Case 2}

A 23 year old farmer from Raipur, India presented with 1 year history of progressive swelling over postero-medial aspect of left thigh and proximal leg (Fig. 7). Swelling was painful and caused hindrance in movement of leg. There was no history of trauma or significant weight loss.

Patient was investigated and a venous color doppler was performed which showed a large lobulated thick walled multiseptated cystic lesion involving posteromedial aspect of lower third of left thigh and upper half of left calf in subcutaneous plane. CT peripheral angiogram showed complex cystic lesion with peripheral enhancement in sartorius and gastrocnemius muscle. MRI left thigh and left leg showed a well defined heterogeneous signal intensity lesion at distal sartorius muscle along with medial compartment of thigh and another similar lesion in superficial plane at popliteal fossa and upper calf region (Fig. 8). FNAC showed only clusters of neutrophils.

Diagnosis was confirmed by truecut core biopsy which showed fibromuscular tissue with numerous variable sized thick walled sporangia containing endospores in a background of chronic mixed inflammatory infiltrate comprising predominantly of lymphocyte and plasma cells. There was no evidence of malignancy in the sections examined. Patient was counseled for surgery and a marginal excision of the mass performed by an extended posterior approach to the knee (Fig. 9). 


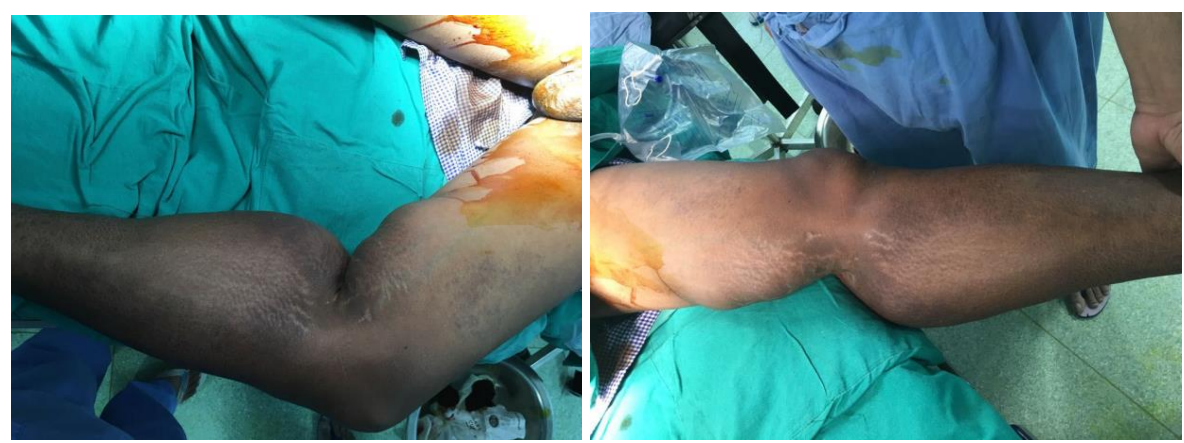

Fig. 7: Swelling over postero-medial aspect of left thigh and proximal leg

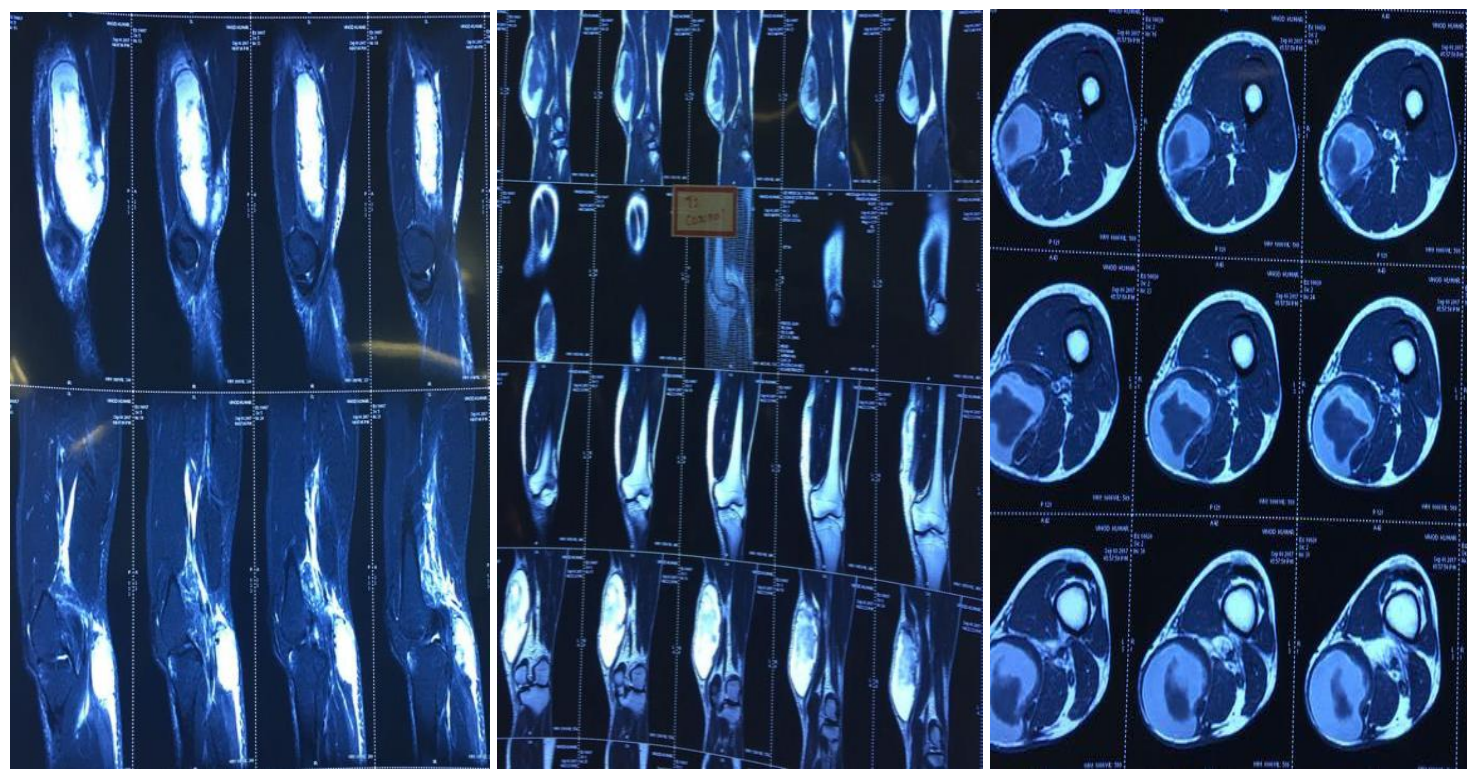

Fig. 8: MRI left thigh and left leg well defined heterogeneous signal intensity lesion noted at distal sartorius muscle
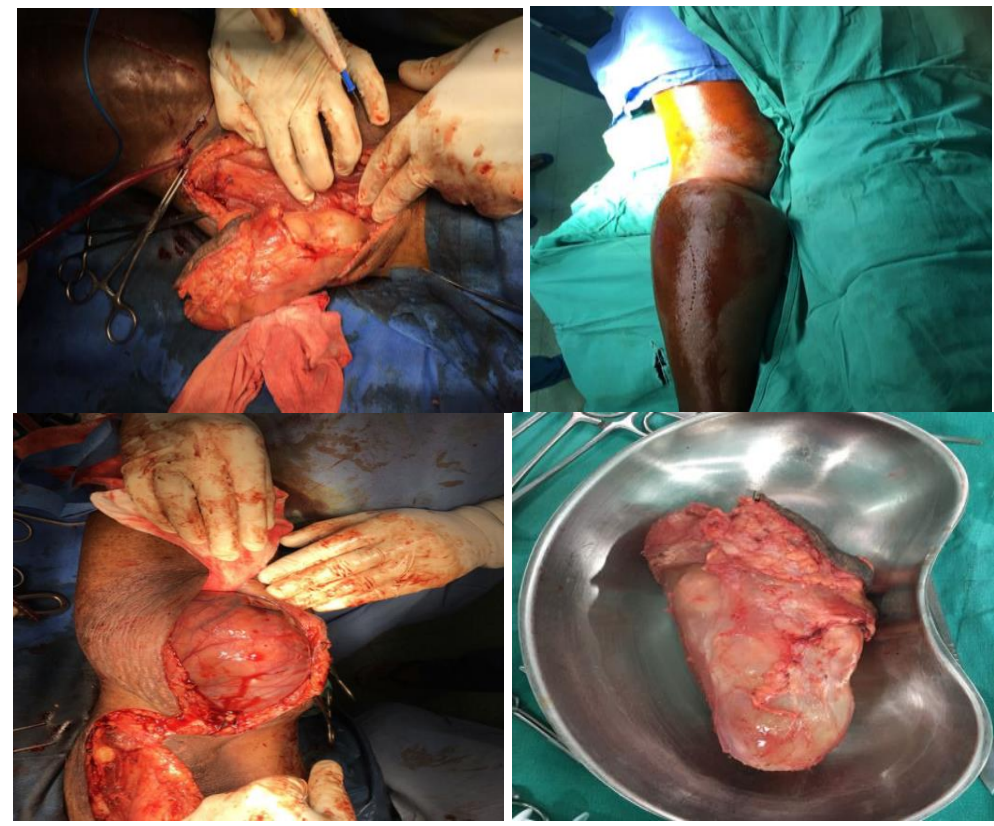

Fig. 9: Marginal excision of the mass 


\section{Case 3}

A 28-year-old farmer, on examination had nontender, hard, fixed, non pulsating swelling of size $10 * 5$ $\mathrm{cm}$ over his right shoulder (Fig. 10) with overlying skin hyperpigmented and ulcerated anteriorly. An expansile, lytic, destructive lesion involving the lateral end of the right clavicle with soft tissue extension was visible on. The clinicoradiological impression was of a primary bone tumor. No mucocutaneous lesions were seen.

Histopathological examination of the lesion revealed many sporangia \& spores with presence of lymphocytes and multinucleated giant cell. PAS stained smear showed many sporangia and spores along with giant cell suggestive of rhinosporidiosis. Isolated involvement of clavicle bone is rare in rhinosporidiosis. Dapsone is the drug in the doses of $100 \mathrm{mg}$ OD for 6 months that is regarded as useful for treating rhinosporidiosis.

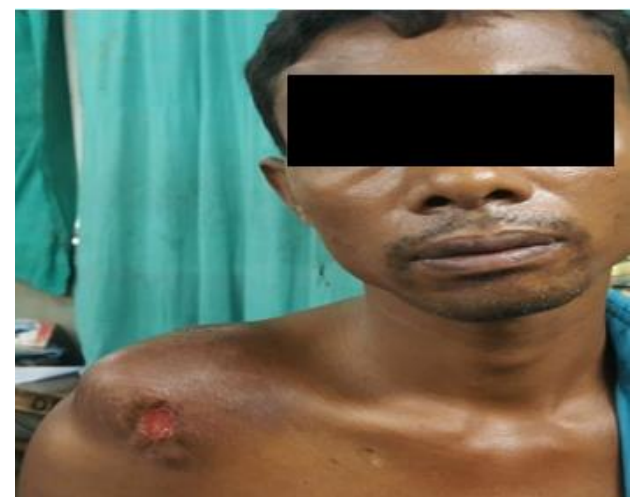

Fig. 10: Clinical picture of swelling over rigth shoulder

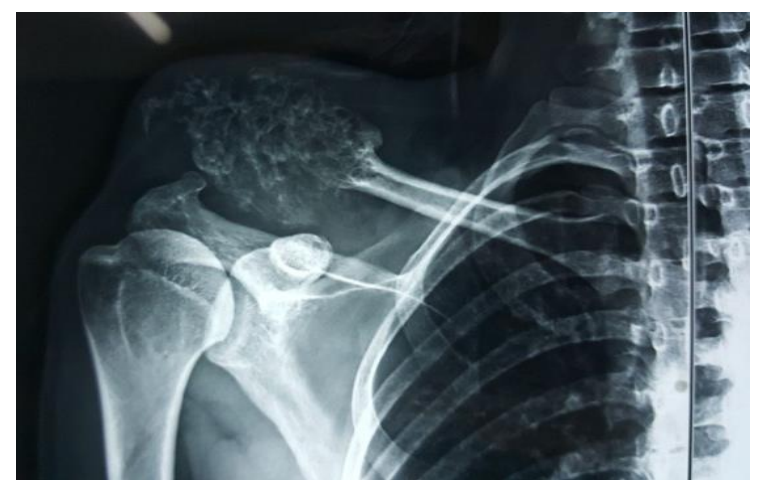

Fig. 11: Radiograph showing destruction involving the of the right clavicle

\section{Discussion}

Rhinosporodiosis seeberi was initially considered a fungus of the sporozoan classification. ${ }^{1}$ However, electron microscopic, histopathological, and molecular studies indicate that it is a eukaryote pathogen rather than a fungus. ${ }^{2}$ It has been recently included into a new class known as Mesomycetozoea. ${ }^{14}$ Rhinosporidiosis has been reported from all over world but the highest incidence has been from Sri Lanka and India. In India,
Chhattisgarh is known to be an endemic area. ${ }^{15} R$ seeberi cannot be isolated in synthetic media in vitro, although it grows well in cell culture. ${ }^{3}$ Its diagnosis depends on the recognition of sporangia and spores in different stages of maturation. Its differential diagnosis includes chondrosarcoma ${ }^{4}$ giant cell tumour, ${ }^{5}$ plasmocytoma, metastasis, and coccidiomycosis. The sporangia of $R$ seeberi should be differentiated from the spherules of Coccidioides immitis. The sporangia of $R$ seeberi are larger and thick walled as well as the size and number of endospores on sporangia of $R$ seeberi are also more than those of $C$ immitis. Rhinosporidiosis is associated with rural and aquatic environments and is transmitted by direct contact with spores through dust, infected clothing or fingers, swimming in stagnant water, trauma, followed by autoinoculation. Hematogenous dissemination is reported to result in painless, firm-to-hard, subcutaneous nodules that remain unattached to skin. ${ }^{6}$ It involves the nasal mucosa in $70 \%$, and eye in about $15 \%$ cases. Visceral involvement, particularly of the liver, lung, and brain has been reported in disseminated disease. ${ }^{7}$ Rhinosporidiosis involving the bone is rare and can manifest in 3 ways- local invasion of skull by nasal rhinosporidiosis, as a part of disseminated rhinosporidiosis, and as a primary manifestation, without any evidence of lesions elsewhere. ${ }^{8}$ Osteolytic lesions involving the tibia, ${ }^{4,9,10}$ lateral condyle femur, ${ }^{1,11}$ hand and feet, ${ }^{8}$ and calcaneus, ${ }^{12}$ have been reported. Dapsone therapy along with surgical excision of the mass with wide margins by diathermy is the treatment of choice. Dapsone ceases the maturation of the spores and accentuation of granulomatous response. ${ }^{13}$

\section{References}

1. Dash A, Satpathy S, Devi K, Das BP, Dash K.

Cytological diagnosis of rhinosporidiosis with skeletal involvement - a case report. Indian J Pathol Microbiol. 2005;48:215-217.

2. Emmons SW, Binfard CH, Utz JP, Kwon-Chung KJ. Medical mycology. 3rd ed. Philadelphia: Lea and Febiger; 1977:464-470.

3. Morelli L, Polce M, Piscioli F, Del Nonno F, Covello R, Brenna A, et al. Human nasal rhinosporidiosis: an Italian case report. Diagn Pathol. 2006;1:25.

4. Aravindan KP, Viswanathan MK, Jose L. Rhinosporidioma of bone: a case report. Indian J Pathol Microbiol 1989;32:312-3

5. Chatterjee PK, Khatua CR, Chatterjee SN, Dastidar N. Recurrent multiple rhinosporidiosis with osteolytic lesions in hand and foot. A case report. J Laryngol Otol. 1977;91:729-734.

6. Ho MS, Tay BK. Disseminated rhinosporidiosis. Ann Acad Med Singapore. 1986;15:80-83.

7. Agrawal S, Sharma KD, Shrivastava JB. Generalized rhinosporidiosis with visceral involvement: report of a case. AMA Arch Derm. 1959;80:22-26.

8. Chatterjee PK, Khatua CR, Chatterjee SN, Dastidar N. Recurrent multiple rhinosporidiosis with osteolytic lesions in hand and foot. A case report. J Laryngol Otol. 1977;91:729-734. 
9. Adiga BK, Singh N, Arora VK, Bhatia A, Jain AK. Rhinosporidiosis. Report of a case with an unusual presentation with bony involvement. Acta Cytol. 1997;41:889-891.

10. Makannavar JH, Chavan SS. Rhinosporidiosis: a clinicopathological study of 34 cases. Indian J Pathol Microbiol. 2001;44:17-21.

11. Gokhale S, Ohri VC, Subramanya H, Reddy PS, Sharma SC. Subcutaneous and osteolytic rhinosporidiosis. Indian J Pathol Microbiol. 1997;40:95-98.

12. Mitra K, Maity PK. Cutaneous rhinosporidiosis. J Indian Med Assoc. 1996;94:84.

13. Job A, Venkateswaran S, Mathan M, Krishnaswami H, Raman R. Medical therapy of rhinosporidiosis with dapsone. J Laryngol Otol. 1993;107:809-812.
14. Herr RA, Ajello L, Taylor JW, Arseculeratne SN, Mendoza L. Phylogenetic analysis of Rhinosporidium seeberi's $18 \mathrm{~S}$ small-subunit ribosomal DNA groups this pathogen among members of the protoctistan Mesomycetozoa clade. J Clin Microbiol. 1999;37:2750 2754.

15. Sudarsan K, Saify AA, Siddique D, Sudarsan V, Agrawal S. Rhinosporidosis of first metatarsal a case report. Indian J Orthop. 1979;13:172-175.

How to cite this article: Kar B. K, Banchhor R, Thakur R.P.S, Mohanty D, Monga N. Rhinosporidiosis with musculoskeletal manifestation: A case series. Indian J Orthop Surg. 2018;4(4):405-410. 